\title{
VIBRATIONS OF A GYROCOPTER - AN ANALYSIS USING IMUS
}

\author{
A. Miraliakbari, M. Hahn and J. Engels \\ Department of Geomatics, Computer Science and Mathematics, University of Applied Sciences Stuttgart, \\ Schellingstraße 24, D-70174 Stuttgart, Germany \\ (alvand.miraliakbari, michael.hahn, johannes.engels)@ hft-stuttgart.de
}

IAG, TC I

KEY WORDS: Aerial, Analysis, IMU, Platforms, Test, Imagery

\begin{abstract}
:
As a part of a research project on the development of a low-cost sensor system for use on gyrocopters several investigations on the recording of aerial image blocks have been carried out during the last two years. The vibration of the gyrocopter platform is one of the critical factors that should be mitigated during the data collection. Apart from the wind-induced oscillation, the vibration induced by the motor, the propeller and the main rotor are prominent. To prevent negative impact to the imaging process, vibration absorbers are to be implemented on the sensor platform.

One focus of this research is a comparison between the vibrations within the passenger area and the vibrations at the undercarriage of the gyrocopter. The comparison is based on the use of three synchronized micro-electro mechanical motion trackers (MEMS IMUs), one fixed on the gyrocopter floor and the others fixed on the struts of the gyrocopter. The results indicate that the propeller and rotorinduced vibrations are somewhat bigger within the passenger area.

Fourier analysis shows that the rotor-induced vibration at a frequency of $12 \mathrm{~Hz}$ is predominant in all signals; its amplitude may vary strongly in time. Whereas the translational vibrations have negligible impact on the image quality, rotational vibrations around all three axes may effect significant blurring of aerial images according to common quality standards.
\end{abstract}

\section{INTRODUCTION}

The typical vehicles used in aerial photography are airplane and helicopter. However, image flights with these vehicles are expensive, which accounts for the current high prices of enduser survey products like ortho-photos and DTMs. Therefore recently Unmanned Airborne Vehicles (UAV) are increasingly applied for photogrammetric purposes. The applicability of UAVs, however, is limited due to legal restrictions, small loading capacity, instable flight characteristics and small operation distances. We think that in this dilemma gyrocopters can represent a notable alternative.

Gyrocopters are lightweight aircrafts which resemble helicopters; in contrast to the latter they gather uplift by a passive rotor which is driven only by the fair wind. A propeller drive is providing the forward motion. Special features of gyrocopters are low acquisition and maintenance costs, low consumption of fuel, high security (there is no breakaway of the airflow if the velocity falls below a certain limit) and a high flexibility in speed and flying height. In a first encouraging experiment (Miraliakbari et al, 2009) we performed a successful image flight with the gyrocopter. In order to adhere consequently to a low-cost approach, we used a simple programmable SLR camera and a MEMS GPS/IMU for the positioning. The recorded images were oriented by an aerial triangulation and a first orthophoto mosaic with $10 \mathrm{~cm}$ ground resolution could be derived.

Whereas the overall image quality was surprisingly good, we have occasionally encountered images with a strong blurring. The probably most important agents of this image blurring are vibrations, which may compromise recordings from a gyrocopter. The movement of the rotor is not completely horizontal. The rotor is connected to the axis by a flapping hinge, allowing a slightly rocking motion which may propagate to a small but noticeable extent to the body of the aircraft. Due to their relatively light weight of 200 to $400 \mathrm{~kg}$, gyrocopters are in addition moderately sensitive for wind excitations. Furthermore, as the space within the gyrocopter is limited, the camera had to be mounted on a strut of the undercarriage, so that it is even more exposed to the fair wind. The induced vibrations do not only generate oscillations in the camera orientation, they may also effect drifts in the IMU sensors, in particular in case of shocks (MicroPilot, 2012). So vibrations may dilute similarly the image quality and the accuracy of the georeferencing.

It is therefore necessary to isolate the camera box or platform, respectively, against occurring vibrations. This is commonly achieved by so-called shock mounts or anti-vibrators, which essentially consist in hydraulic (e.g. oil-filled) or solid springs. In order to achieve a sufficient isolation of the sensor platform, suitable shock mounts are dimensioned depending on the payload of the system and also the characteristics of the vibration. Protection against individual shocks has to be distinguished from protection against proper (periodic) vibrations. Whereas for the former task high spring constants are suitable in order to attenuate the instantaneous shocks, for the latter task the expected excitation frequency is the decisive parameter in the system design. Badly selected spring parameters may even amplify the vibrations, viz. if an eigenfrequency of the shock mount system coincides with an excitation frequency. An instructive introduction to the design of shock mounts is given in (MicroPilot , 2012). In any case, in order to achieve an effective isolation, it is of crucial importance to select the parameters of the shock mounts carefully, depending on the expected excitation.

A lot of research has been undertaken to investigate the vibrations of aircrafts. Frequently vibrations are artificially excited: ground vibration tests are standard for health 
monitoring of aerospace structures, i.e. in order to prevent failures due to material fatigue (Pickrel et al, 2006). Similarly the modal behavior of aircrafts is studied (Peeters et al, 2008). Main target of our present paper is to investigate the vibrations occurring at the undercarriage and the passenger area of a gyrocopter, in view of image blurring. The result should serve as a basis for the design of an appropriate shock mount system.

In general high frequency excitations are easier to isolate than low frequency excitations. On the other hand, if the period of a vibration is much higher than the respective exposure time of the camera, it will effect less image blurring than a high frequency vibration with the same amplitude, because during the exposure interval only a part of the amplitude range is covered. Several authors have studied the impact of vibrations on aerial imagery; it has been evaluated e.g. in terms of the Modulation Transfer Function (MTF) or in terms of blur radii (Trott, 1960), (Wulich and Kopeika 1987). Here we abstain from a through consideration of the mentioned blurring measures and rather describe the blurring effect by the displacement on ground which is caused by rates of turn around $\mathrm{X}, \mathrm{Y}$ and $\mathrm{Z}$ axis.

\section{HARDWARE AND SOFTWARE}

The gyrocopter used in our research belongs to the MTOsport series. It is a gyro plane with nose gear wheel chassis. There are two seats for the pilot and the passenger. The rotor is made of aluminum; rudder and the stabilizer surface are produced from GPR (or carbon fiber). The length, width and height are $5.08 \mathrm{~m}$, $1.88 \mathrm{~m}$ and $2.71 \mathrm{~m}$, respectively. The empty weight of this kind of gyrocopter is $240.8 \mathrm{~kg}$ to $247.0 \mathrm{~kg}$. The payload is $203.0 \mathrm{~kg}$ to $209.2 \mathrm{~kg}$. Fuel tank capacity is $34 \mathrm{ltr}$, which can be increased by means of an auxiliary fuel tank up to 68 ltr. (MTOsport, 2011).

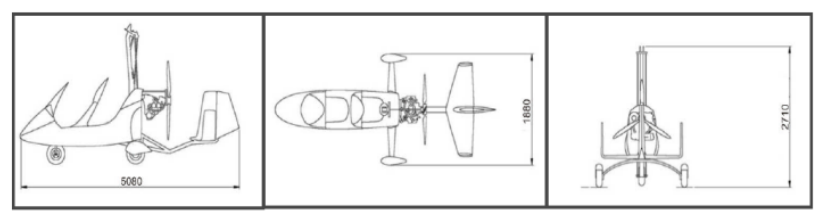

Figure 1. Three views of MTOsport (MTOsport, 2011)

Apart from the wind-induced oscillations, the vibrations induced by the motor, the propeller and by the main rotor are prominent, see Table 1 for the typical frequencies.

\begin{tabular}{|c|c|c|}
\hline Excitation & $\begin{array}{l}\text { Rotational } \\
\text { frequencies(Hz) }\end{array}$ & $\begin{array}{l}\text { Induced vibrational } \\
\text { frequencies }(\mathrm{Hz})\end{array}$ \\
\hline Motor & $75-95$ & \\
\hline Propeller & $30-40$ & $90-120$ \\
\hline Rotor & $5.8-6.7$ & $11.6-13.4$ \\
\hline
\end{tabular}

Table 1. Typical vibrational frequencies induced by motor, propeller and rotor

The propeller is gear-reduced with a factor of 2.43 , therefore the propeller frequency is lower than the motor frequency by this factor. In general the induced vibrational frequencies differ from the excitation frequencies: As the propeller features three blades, its induced vibration frequency is three times higher than the propeller frequency itself. The rotor has two blades which yields a frequency doubling.

Accelerations and gyro rates were observed with multiple MEMS GPS/IMUs of type MTi-G, manufactured by Xsens Company (Xsens, 2009). The MTI-G is a small size and low weight sensor and therefore highly suited for the positioning in unmanned and small size aerial vehicles. It provides different filter settings and constraints, combined in "scenarios". The user can select the respective scenario depending on the type of the application (Xsens, 2012a). Table 2 lists the manufacturer information about the accuracy of MTi-G in position and attitude.

\begin{tabular}{|l|c|}
\hline Static accuracy (roll/pitch) & $<0.5 \mathrm{deg}$ \\
Static accuracy (heading) & $<1 \mathrm{deg}$ \\
Dynamic accuracy & $1 \mathrm{deg} \mathrm{RMS}$ \\
Angular resolution & $0.05 \mathrm{deg}$ \\
Accuracy of position & $2.5 \mathrm{~m}$ \\
\hline
\end{tabular}

Table 2. Accuracy of MTi-G in position and attitude. (Xsens, 2012b)

Figures 2 and 3 show the position of IMUs on the undercarriage of the gyrocopter and in the foot space of the cabin, respectively.

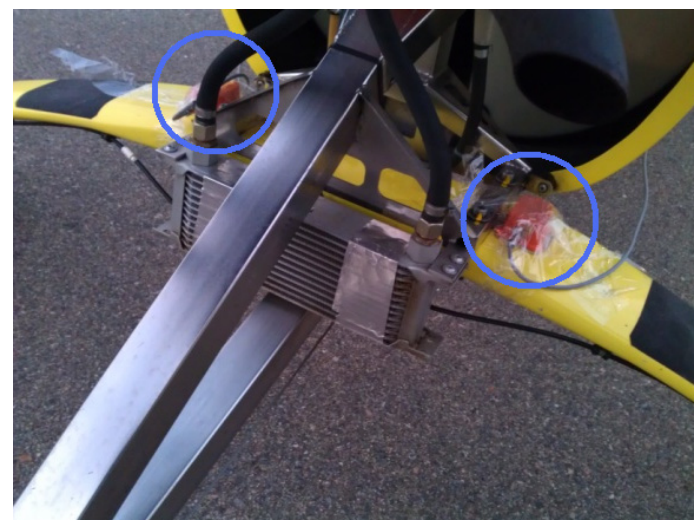

Figure 2. Position of two IMUs shown within the blue circles.

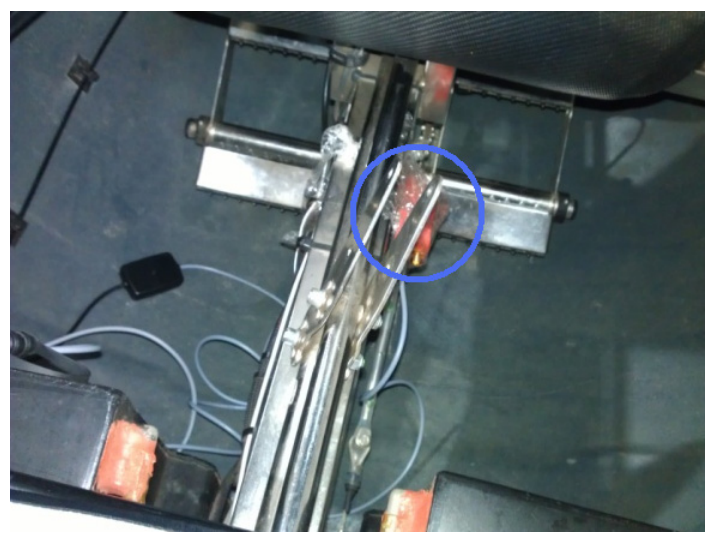

Figure 3. Position of the last IMU shown within the blue circle. 


\section{TIME-FREQUENCY ANALYSIS}

In most of our experiments the position and orientation parameters could be represented well by distinct stationary oscillations. In such cases a Discrete Fourier Transform (DFT) is the adequate tool for the analysis. The DFT is computed according to

$$
\widehat{\bar{f}}(n \Delta v)=c_{n}=\frac{1}{N} \sum_{k=0}^{N-1} f(k \Delta t) \exp \left(-i \frac{2 \pi}{N} k n\right)
$$

Here $N$ denotes the number of observations within the sample, $\Delta \boldsymbol{v}$ is the basic frequency of the interval, $c_{n}$ the Fourier coefficient.

Our sampling frequency was usually $150 \mathrm{~Hz}$, so the resulting Nyquist frequency of $75 \mathrm{~Hz}$ is evidently lower than the highest expected vibrational frequencies which are induced by the motor and the propeller, see Table 1 . We have therefore to be aware of possible aliasing effects. The aliasing can be modelled according to the well-known rule (Keller, 2004)

$$
\widehat{\bar{f}}(v)=\hat{f}(v)+\hat{f}\left(v+v_{s}\right)+\hat{f}\left(v-v_{s}\right)
$$

Here $\boldsymbol{v}_{\mathrm{S}}$ denotes the sampling frequency. $\hat{f}(v)$ is the spectrum of the actual signal and $\hat{\bar{f}}(v)$ the aliased spectrum. Let $v_{E}$ be the frequency of the high-frequent aliasing signal. The contaminated frequencies are therefore

$$
v=v_{E} \pm v_{S}
$$

In our case $\boldsymbol{v}_{\mathrm{E}}$ varies in the range of 90-120 Hz. We may therefore expect that the frequencies between 30 and $60 \mathrm{~Hz}$ are contaminated.

As the phase angles are of marginal interest here, we consider mostly the square root of the energy spectrum of the respective signal. The corresponding amplitudes feature the same dimension as the original signal; thus they permit a straightforward assessment of the effected motion blurring in the aerial images.

For some data samples the vibrations manifested some nonstationarities due to irregular wind blasts or compensating aircraft activities. Non-stationarities during the gyrocopter's takeoff phase are not our concern, as the registration of the images happens in stable straight-ahead flight. For investigations of non-stationary effects a wavelet analysis suggests itself. The continuous wavelet transform is defined by

$$
\begin{aligned}
W\{f\}(\lambda, t):= & \frac{1}{\sqrt{c_{\psi}}}|\lambda|^{-\frac{1}{2}} \int_{-\infty}^{\infty} f(u) \psi\left(\frac{u-t}{\lambda}\right) d u, \\
& \lambda \in R \backslash\{O\}, t \in R
\end{aligned}
$$

with

$$
c_{\psi}:=2 \pi \int_{-\infty}^{\infty} \frac{|\widehat{\psi}(\omega)|^{2}}{\omega} d \omega
$$

Here $c_{\psi}$ denotes the admissibility constant, $\psi$ denotes the analysing wavelet (Keller, 2004). Here we use the well-known Morlet-wavelet, (Goupillaud et al, 1984) and (Schmidt, 2001) as it features good localization properties in time and frequency space. It is defined as:

$$
\psi_{M}(s):=\exp \left(i \kappa_{0} s\right) \cdot\left(\exp \left(-\frac{s^{2}}{2 \sigma^{2}}\right)-\sqrt{2} \exp \left(-\frac{\kappa_{0} \sigma^{2}}{4}\right) \cdot \exp \left(-\frac{s^{2}}{\sigma^{2}}\right)\right)
$$

The Morlet-wavelet equals in good approximation a harmonic oscillation modulated by a Gaussian; the parameter $\kappa_{0}$ corresponds to the angular frequency of the oscillation, $\sigma$ is the spread of the Gaussian envelope. Here we set $\kappa_{0}=2 \pi$; for this choice the frequency of the wavelet is equal to 1 , and the scale factor $\lambda$ can be directly interpreted as period or inverse frequency of the wavelet transform. The choice of a big value for $\sigma$ yields good frequency localization, whereas small values for $\sigma$ favour a good representation of non-stationarities of the signal.

A wavelet transform according to Formula (4) can be interpreted as a parameterized family of linear filters having the scale $\lambda$ as parameter. If the wavelet is sufficiently localizing in frequency space, the filter characteristic for an arbitrary, but fixed $\lambda$ is quite narrow. Thus the result of the wavelet transform for fixed $\lambda$ is essentially a (slightly modulated) oscillation; its frequency is given by the central frequency of the wavelet divided by $\lambda$. For an interpretation of the result, however, it is more convenient to consider the amplitudes of those oscillations than the oscillations themselves. The corresponding transform is called wavelet scalogram. As the Morlet-wavelet constitutes a complex wavelet, the scalogram is simply obtained as the modulus of the complex wavelet transform. Numerically the wavelet transform is computed by an application of the convolution theorem of Fourier analysis. It is obvious that the aliasing problem is affecting the Wavelet transform similarly to the Fourier analysis.

The application of wavelet transforms in vibration analysis is discussed in detail in Lepik (2001). Smith et al. (2007) have used wavelet transforms to analyze vibration for the purpose of aircraft health monitoring.

\section{EXPERIMENTAL INVESTIGATION AND RESULTS}

We performed two test flights in order to investigate the vibrational behaviour of our imaging system. We have used up to three synchronized IMUs. In principle the Xsens MTi-G IMUs allow sampling rates up to $512 \mathrm{~Hz}$. Due to problems related to the baud-rate compatibility of the PC interface, we were limited to $150 \mathrm{~Hz}$ or, if GPS data were to be stored, even to $120 \mathrm{~Hz}$.

\subsection{Test Flight with three Synchronized IMUs}

In a first flight, we have used three IMUs; two of these were mounted on the struts of the undercarriage of the gyrocopter (left and right), the third IMU was fixed in the foot space inside the gyrocopter. Figures 2 and 3 show the positions of the IMUs. The respective flight was performed in order to test the synchronization and to assess the dependency of the vibration on the exact mounting position on the gyrocopter.

For the analysis we use a sequence of 1024 observations which corresponds to a period of just 7 seconds of a stable flight. A comparison of the results from the individual IMUs indicates a good agreement. Figure 4 shows the square roots of the energy spectra for the acceleration in flight direction. In exceptional cases the differences between the square roots of the energy spectra can reach $0.2 \mathrm{~m} / \mathrm{s}^{2}, 0.4 \mathrm{~m} / \mathrm{s}^{2}$ and $0.6 \mathrm{~m} / \mathrm{s}^{2}$ for acceleration in flight direction, across flight direction and in vertical direction, respectively. The corresponding differences for the angular rates around flight direction, across flight direction and vertical direction reach $0.02 \mathrm{rad} / \mathrm{s}, 0.01 \mathrm{rad} / \mathrm{s}$ and $0.02 \mathrm{rad} / \mathrm{s}$, respectively. 

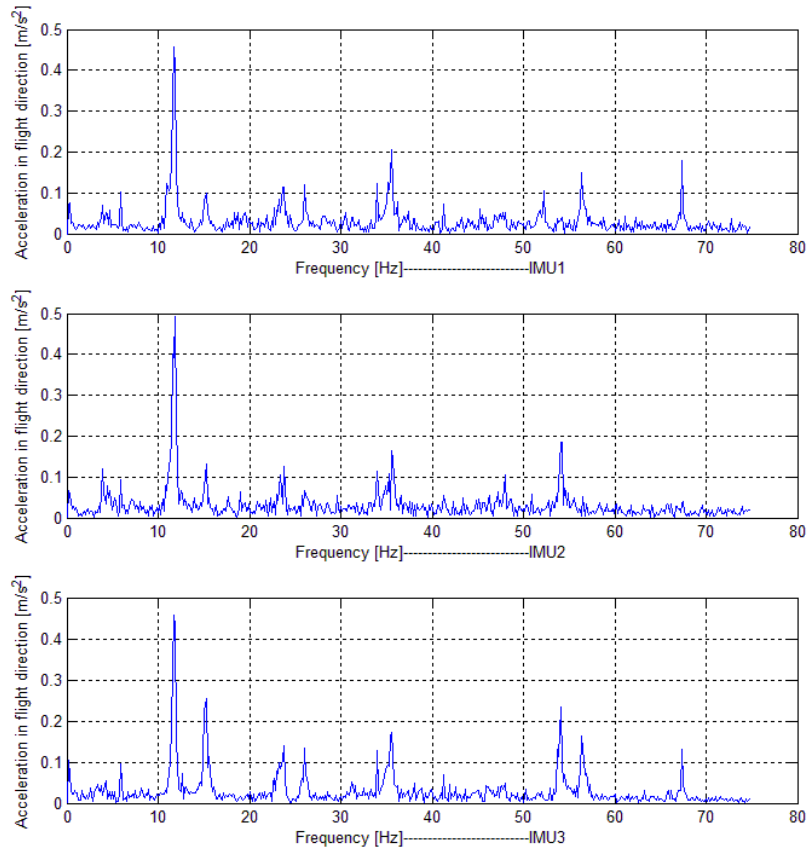

Figure 4. Comparison of FFT results for acceleration in flight direction (uppermost: left strut, middle: right strut and lowermost: inside the passenger area)

Some exceptional differences indicate that the vibrations at the IMU inside the gyrocopter were slightly stronger than on the struts of the undercarriage. This might be due to the fact that the internal IMU was fixed very close to the metallic bearing structure of the gyrocopter, which can be expected to conduct the vibrations of the rotor and the propeller best.

\subsection{Test Flight with one IMU}

In this test only one IMU was mounted on the left strut of the gyrocopter. The sampling frequency was the same as in the previous test using three IMU's $(150 \mathrm{~Hz})$. For the analysis a sequence of around 30000 observations (3.5 min) was selected As in the previous test we have analyzed 6 different observables viz. $3 \mathrm{D}$ accelerations and rates of turn around the $\mathrm{X}, \mathrm{Y}$ and $\mathrm{Z}$ directions.

Figure 5 shows histograms of the observed values, from which it becomes evident that the vibrations can seriously affect the image quality. For the rate of turn around the flight direction $10 \%$ of the absolute values exceed $0.1 \mathrm{rad} / \mathrm{s}$. With a flying height of $500 \mathrm{~m}$ and an exposure time of $0.008 \mathrm{~s}$ this corresponds to a maximal ground displacement of $0.44 \mathrm{~m}$, which is for typical ground pixel sizes of 10 to $20 \mathrm{~cm}$ clearly intolerable.

The rates of turn around the $\mathrm{Y}$ axis exceed $0.03 \mathrm{rad} / \mathrm{s}$ for about $10 \%$ of observed values. With the same integration time and flight height as above, the displacement is about $0.12 \mathrm{~m}$ on the ground.

The rates of turn around the $\mathrm{Z}$ axis exceed $0.07 \mathrm{rad} / \mathrm{s}$ for about $10 \%$ of observed values. If we assume the ground coverage of the image to be $500 \mathrm{~m}$ in width, the corresponding rotation yields $0.14 \mathrm{~m}$ displacement at the boundary of the imaged area. On the other hand, by integration of the accelerations in all three directions it becomes obvious that the vibration-induced translational velocities are much smaller than the flight velocity, hence the translational vibrations do no harm to the registered images.
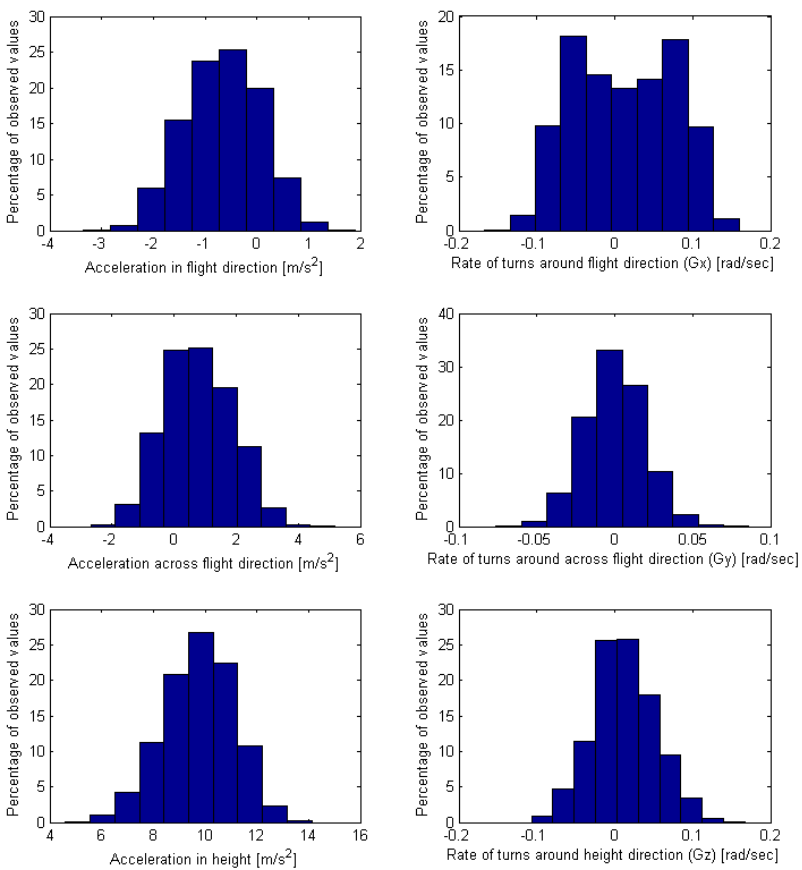

Figure 5. Comparison of histograms of the observed results for accelerations and rates of turn

\subsubsection{FFT Transforms}

As already discussed in the introduction, the isolation against vibration has to be designed in compliance with the expected excitation frequencies. Therefore Fourier transforms of the observed time series have been computed in order to explore the characteristic frequencies.

In spite of the relative long observation period, the energy spectra feature quite distinct peaks, hence indicate a stationary behavior of the respective signal.
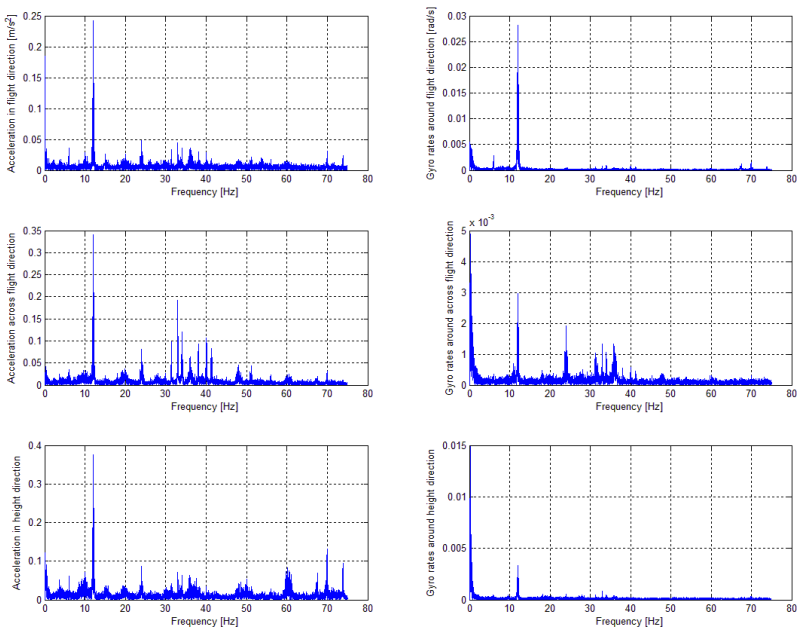

Figure 6. Square root of the energy spectra of accelerations (left column) and rates of turn (right column) related to X, Y, Z

For all observables we encountered high energy at very low frequencies $(0-2 \mathrm{~Hz})$, which are most probably induced by wind or adjusting flying movements. Most prominent in all cases is the peak at $12 \mathrm{~Hz}$ (Figure 6), which is evidently induced by the rotor, compare Table 1. Less pronounced, but still significant at 
least for the accelerations is a sequence of peaks in the range of 25 to $50 \mathrm{~Hz}$ and in particular in the height direction above 60 $\mathrm{Hz}$. The vibration energy in the former range might originate to a big extent from propeller vibrations; as discussed in section 3, aliasing has to be expected in the range between 30 and $60 \mathrm{~Hz}$.

The vibration period corresponding to $12 \mathrm{~Hz}$ is big compared with the common exposure times $(1 / 100 \mathrm{~s}-1 / 500 \mathrm{~s})$. As we are considering the rates of turn in our calculations, not the angles themselves, we may just multiply the amplitudes with the exposure times in order to obtain maximal incremental angles. The rotor vibration is the one with the biggest impact on the image quality. The maximum amplitude of almost $0.03 \mathrm{rad} / \mathrm{s}$ around the $\mathrm{X}$ axis, amounts to $1 / 3$ of the $10 \%$ quantile observed in the histogram for the rates of turn around $\mathrm{X}$ (figure 5).

Figure 6 shows also some energy in the very low frequency range between 0 and $1 \mathrm{~Hz}$ which can hardly be isolated by the vibration absorbers.

\subsubsection{Wavelet Transform}

For the retrieval of possible instationarities in the signals a wavelet analysis is helpful. Here we perform a CWT with the Morlet wavelet. Figures 7 and 8 represent the wavelet scalograms related to acceleration in flight direction and also the rates of turn around the flight direction. Because of the long sampling period, only the first 20 seconds of the scalograms are displayed. Here $\sigma=1$ and $\mathrm{k}=2 \pi$ have been selected as parameters, which corresponds to a good localization in the time domain.

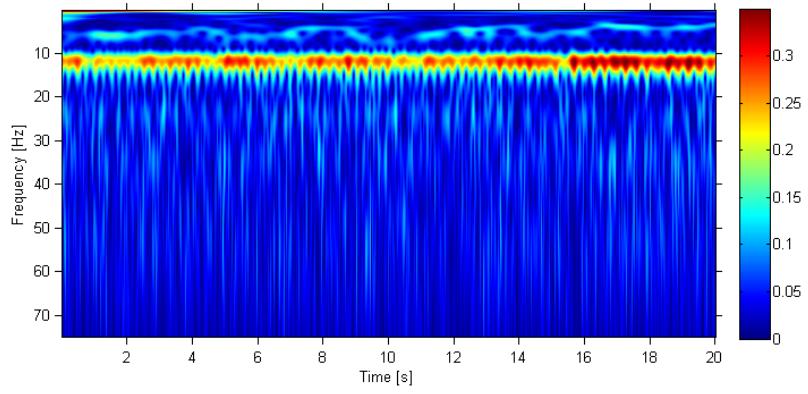

Figure 7. Morlet-scalogram of the acceleration in flight direction (first $20 \mathrm{~s}$ )

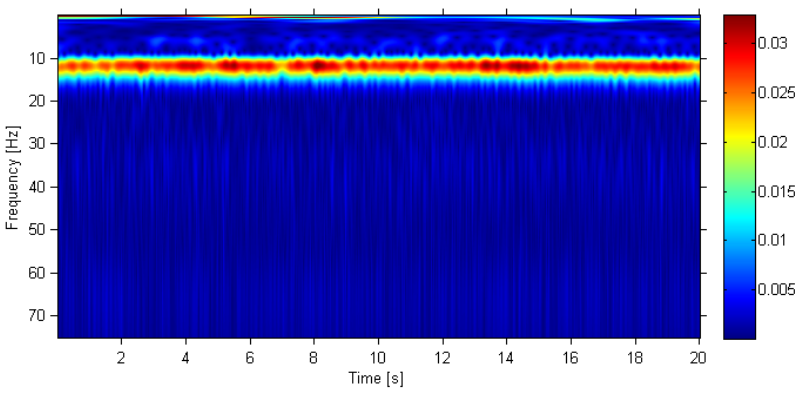

Figure 8. Morlet-scalogram of the rates of turn around the flight direction (first $20 \mathrm{~s}$ )

Anyway the scalogram shows a relative uniform behaviour along the time axis, at least what refers to the frequency values of the maximal amplitudes. The absolute values are not easily interpretable due to some spreading in the frequency direction. As expected, the rotor-induced vibration at $12 \mathrm{~Hz}$ features the highest amplitudes again (mixed red-yellow lines in both scalograms).

Quite interesting are the profiles of the time-dependent amplitude at the fixed frequency of $12 \mathrm{~Hz}$. It becomes evident that these amplitudes vary by ca. $30 \%$ for the acceleration in flight directions and ca. $15 \%$ for the rates of turn around the flight direction.

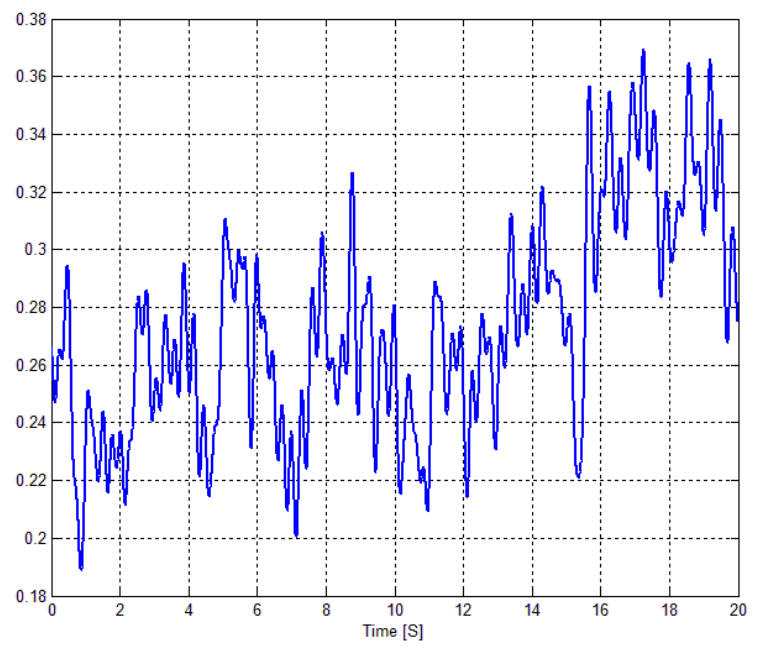

Figure 9. The profile of wavelet scalogram related to acceleration in flight direction at $12 \mathrm{~Hz}$

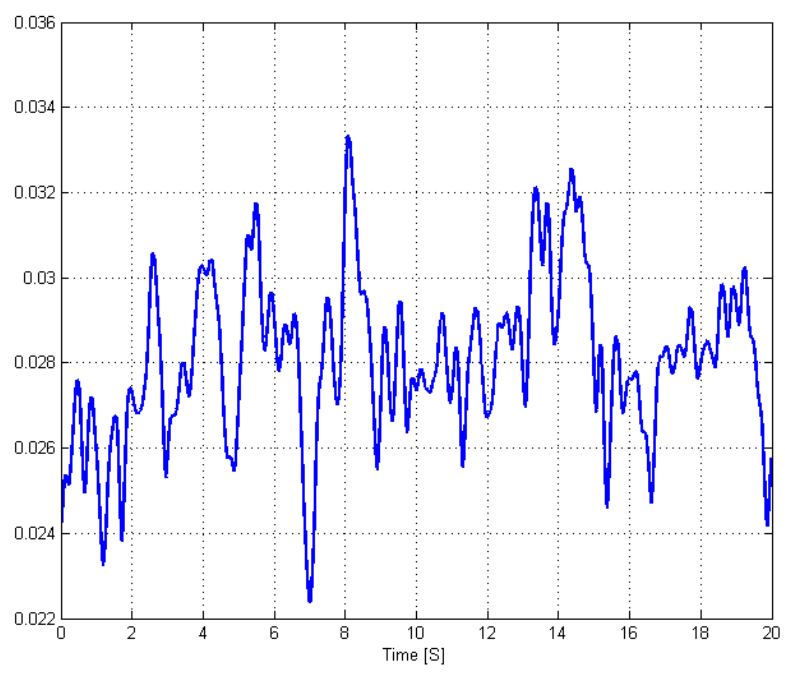

Figure10. The profile of wavelet scalogram related to rates of turn around flight direction at $12 \mathrm{~Hz}$

\section{CONCLUSIONS}

Investigations of vibrations on a gyrocopter have been carried out by observing 3D accelerations and rotational rates around the X-, Y-, and Z axis with up to three IMUs (MTi-G) of the Xsens Company. 
The analysis with the DFT has shown a certain dependency on the locations where the IMUs have been mounted. Surprisingly higher amplitudes have been encountered within the passenger area than at the undercarriage of the gyrocopter. The differences in the accelerations amounted up to $0.6 \mathrm{~m} / \mathrm{s}^{2}$ and in the rates of turn up to $0.02 \mathrm{rad} / \mathrm{s}$. Most prominent in the Fourier transform of all signals is a peak at the frequency of $12 \mathrm{~Hz}$ which can be identified as the doubled rotor frequency. Suitable vibration absorbers essentially have to cope with vibrations of this frequency to prevent negative impact to the imaging process. Wavelet analysis indicated that the $12 \mathrm{~Hz}$ frequency is relatively stable, that however the amplitudes vary significantly over time. Biggest impact on the image data capture has the rate of turn around the flight direction. $10 \%$ of the values exceed $0.1 \mathrm{rad} / \mathrm{s}$ which corresponds to $0.44 \mathrm{~m}$ on the ground assuming a flying height of $500 \mathrm{~m}$ and an exposure time of $0.008 \mathrm{~s}$.

\section{REFERENCES}

Goupillaud, P., Grossmann, A. and Morlet, J., 1984. CycleOctave and Related Transforms in Seismic Signal Analysis. Geoexploration, vol 23, pp. 85-102

Keller, W., 2004. Wavelets in Geodesy and Geodynamics. Walter de Gruyter, Berlin, New York.

Lepik, Ü., 2001. Application of Wavelet Transform Techniques to Vibration Analysis. Institute of Applied Mathematics, University of Tartu.

http://www.kirj.ee/public/va_fm/f-50-3-5.pdf (01 Apr. 2012)

Miraliakbari, A., Hahn, M. and Engels, J., 2009. Aerial survey with a gyrocopter, Applied Geoinformatics for Society and Environment, Stuttgart Germany.

MicroPilot, 2012. Isolating Components from UAV Vibration, Newsletter MicroPilot. Stony Mountain, Manitoba, Canada R0C 3 A0.

http://www.micropilot.com/pdf/isolating-components-uavvibration.pdf (25 Apr. 2012).

MTOsport 2011. Flight and Operation Manual for Gyroplane,. AutoGyro_FOM_MTOsport, Revision 5.0 - Issue Date 01.04.2011.

http://www.us.auto-gyro.com/pdf/FlightManual_MTOsport_50_ENG.pdf (05 Apr. 2012)

Peeters, B., Climent, H., Diego, R., Alba, J., Ahlquist, J., Carreño, J., Hendricx, W., Rega, A., García, G., Deweer, J., Debille, J., 2008. Modern Solutions for Ground Vibration Testing of Large Aircraft.

http://www.irisa.fr/sisthem/flite/ARTICLES/LMS/2008_IMAC _GVT.pdf (20 Apr. 2012)

Pickrel, C., Foss, G., Phillips, A., Allemang, R. and Brown, D., 2006. New Concepts in Aircraft Ground Vibration Testing. Boeing Commercial Airplane Group, Seattle, Washington,

University of Cincinnati, Cincinnati, Ohio.

www.sandv.com/downloads/0610pick.pdf (20 Apr. 2012).

Schmidt, M., 2001. Grundprinzipien der Wavelet-Analyse und Anwendungen in der Geodaesie. Shaker, Aachen.

Smith C., Akujuobi, C., Hamory, P. and Kloesel, K., 2007. An approach to vibration analysis using wavelets in an application of aircraft health monitoring. Mechanical Systems and Signal Processing, Vol 21, Issue 3, pp 1255-1272

Trott, T., 1960. The effects of motion in resolution. Photogrammetric Engineering 26, pp. $819-827$

Wulich, D. and Kopeika, N., Image resolution limits resulting from mechanical vibrations. Optical Engineering 26, 529-533 (1987).

Xsens, 2009. MTi- G user manual and technical documentation. XSens Technologies B.V. Document MT10137P, revision G, 27 May 2009.

Xsens, 2012a. MTi-G Overview. Xsens Technologies, B.V. http://www.xsens.com/en/general/mti-g, (02 Apr .2012).

Xsens, 2012b. MTi-G leaflet. Xsens Technologies, B.V. http://www.xsens.com/images/stories/products/PDF_Brochures/ mti-g\%20leaflet.pdf (04 Apr.2012)

\section{ACKNOWLEDGEMENT}

The authors thank Pascal Bouygues (Flugschule Gyrocopter Stuttgart-München) for his support throughout this research.

This work was funded by the Ministry of Science, Research and Arts of the state of Baden-Württemberg, Germany within the project GyroTIR. 